\title{
Double layer acceleration by laser radiation
}

\author{
SHALOM ELIEZER, ${ }^{1,2}$ NOAZ NISSIM, ${ }^{2}$ JOSÉ MARIA MARTÍNEZ-VAL, ${ }^{1}$ KUNIOKI MIMA, ${ }^{3}$ AND \\ HEINRICH HORA ${ }^{4}$ \\ ${ }^{1}$ Institute of Nuclear Fusion, Polytechnic University of Madrid, Madrid, Spain \\ ${ }^{2}$ Soreq Research Center, Yavne, Israel \\ ${ }^{3}$ Institute of Laser Engineering, Osaka University, Osaka, Japan \\ ${ }^{4}$ Department of Theoretical Physics, University of New South Wales, Sydney, Australia
}

(RECEIVED 30 October 2013; ACCEPTED 13 November 2013)

\begin{abstract}
It is shown that it is possible to accelerate micro-foils to velocities from $10^{8} \mathrm{~cm} / \mathrm{s}$ up to relativistic velocities without the disturbance of the Rayleigh-Taylor instability. The acceleration occurs due to the radiation pressure of proper high power lasers. In these systems, the ablation force is negligible relative to the ponderomotive force that dominates the acceleration. The laser irradiances of $10^{17} \mathrm{~W} / \mathrm{cm}^{2}<I_{L}<10^{21} \mathrm{~W} / \mathrm{cm}^{2}$ with a pulse duration of the order of 10 picoseconds can accelerate a micro-foil by the laser radiation pressure to velocities as high as $10^{9} \mathrm{~cm} / \mathrm{s}$ before breaking by Rayleigh Taylor (RT) instability. Similarly, laser irradiances of $I_{L}>10^{21} \mathrm{~W} / \mathrm{cm}^{2}$ with pulse duration of the order of 10 femtoseconds can accelerate a micro-foil to relativistic velocities without RT breaking. Due to the nature of the accelerating ponderomotive force, in both the relativistic and non-relativistic cases, the structure of the accelerated target contains a double layer (DL) at the interface of the laser-target interaction. The DL acts as a piston during the acceleration process. The influence of the DL surface tension on the RT instability is also analyzed in this paper.
\end{abstract}

Keywords: Foil acceleration; High intensity laser; Plasma; Rayleigh-Taylor instability

\section{INTRODUCTION}

The use of spherical shells in inertial confinement fusion, in comparison with solid spheres, has reduced significantly the peak laser power and energy required to ignite a target of a given mass. The ablation pressure $P_{a}$ that accelerates the shell is a function of the irradiating laser intensity $I_{L}$ which scales approximately like $P_{a} \sim I_{L}^{2 / 3}$. For a shell with initial radius $R_{0}$ and thickness $\Delta R_{0}$, the implosion velocity $v_{f}$ scales as $v_{f}^{2} \sim P_{a}\left(R_{0} / \Delta R_{0}\right)$. The resulting stagnation pressure is $P_{f} \sim P_{a}\left(R_{0} / \Delta R_{0}\right) G$, where $G$ is a radial convergence factor of the order of 100 . The minimum energy of ignition scales as $1 / P_{f}^{2}$. In order to get ignition, the ablation pressure of about $30 \mathrm{Mbars}$ has to increase to $P_{f} \sim 100 \mathrm{Gbars}$, i.e., by a factor of 3000, implying an aspect ratio $R_{0} / \Delta R_{0} \sim 30$. The larger the aspect ratio is the higher the implosion pressure $\left(P_{f}\right)$ one gets implying smaller laser energy $(\sim 1 /$ $\left.P_{f}^{2}\right)$. However, the magnitude of the aspect ratio is limited by Rayleigh Taylor (RT) instability (Rayleigh, 1876; Taylor, 1950). The RT instability can cause a disturbance

Address correspondence and reprint requests to: S. Eliezer, Institute of Nuclear Fusion, Polytechnic University of Madrid, Madrid, Spain. E-mail: shalom.eliezer@gmail.com to grow from extremely small amplitudes to large levels that can break the accelerated foil or the shell before it reaches the desired final velocity. Since the beginning of the inertial confinement fusion program the RT instability has been a subject of uncertainties and controversies.

The highest laser-induced pressures, of the order of $1 \mathrm{Gbar}$, have been obtained during the collision of a target with an accelerating foil. This acceleration was achieved by the ablation of laser produced plasma or by X-rays created by laser plasma interaction. The foil absorbs the laser energy, plasma is then obtained and the foil is accelerated like a rocket (Eliezer, 2002). In this way, the flyer stores kinetic energy from the laser during the acceleration period and delivers it during the collision with a target, in the form of thermal energy. The laser driven flyer can achieve much higher pressures on impact than the shock wave pressure obtained directly by the laser ablation. In 1994, at the Livermore Laboratory, a pressure of about $1 \mathrm{Gbar}$ was created by accelerating a foil with soft $\mathrm{X}$-rays from the indirect drive (Cauble et al., 1993). Planar foil targets were accelerated to $1000 \mathrm{~km} / \mathrm{s}$ at the Institute of Laser Engineering at Osaka University (Azechi et al., 2009). The targets were about $20 \mu \mathrm{m}$ thick and were made of polystyrene doped with 
$0.4 \%$ (atomic) bromine. The acceleration driver was a $0.35 \mu \mathrm{m}$ wavelength laser with energy of $1.5 \mathrm{~kJ}, 2 \mathrm{~ns}$ pulse width and an irradiation of $4 \times 10^{14} \mathrm{~W} / \mathrm{cm}^{2}$.

For $I_{L}<10^{16} \mathrm{~W} / \mathrm{cm}^{2}$, the ablation pressure is dominant. For $I_{L} \gg 10^{16} \mathrm{~W} / \mathrm{cm}^{2}$, the radiation pressure is the dominant pressure at the solid-vacuum interface and the ablation pressure is negligible. For $I_{L}>10^{21} \mathrm{~W} / \mathrm{cm}^{2}$, it was suggested (Esirkepov et al., 2004; Eliezer 2012) that relativistic acceleration can be achieved.

We are interested in the classical acceleration of a microfoil that can be achieved for laser irradiances in the domain $10^{17} \mathrm{~W} / \mathrm{cm}^{2}<I_{L}<10^{21} \mathrm{~W} / \mathrm{cm}^{2}$ with laser pulses of the order of picosecond where the ablation pressure can be neglected relative to the radiation pressure. We also discuss the relativistic acceleration that can be achieved with $I_{L}>$ $10^{21} \mathrm{~W} / \mathrm{cm}^{2}$.

This paper analyzes the foil acceleration by ponderomotive force. In this model, the force acts on the electrons that are accelerated and the ions that follow accordingly. The system of the negative and positive layers is called a double layer (DL). The DL is regions of non-neutral plasma which induce a large potential drop thereby causing very strong electric fields. In our case, we have DL acceleration by the laser irradiance (Clark et al., 1985; Eliezer \& Hora, 1989; Hora et al., 1984).

In Section 2, the classical laser pressure acceleration and the hydrodynamic Rayleigh-Taylor (RT) instability are calculated relevant to laser irradiances in the domain $10^{17} \mathrm{~W} / \mathrm{cm}^{2}<I_{L}<10^{21} \mathrm{~W} / \mathrm{cm}^{2}$. Section 3 analyzes the surface tension contribution to the RT instability. The relativistic hydrodynamic RT instability appropriate for $I_{L} \geq$ $10^{21} \mathrm{~W} / \mathrm{cm}^{2}$ is summarized in Section 4 . The analysis is discussed in the last section.

\section{LASER PRESSURE ACCELERATION AND RT INSTABILITY}

The radiation pressure $P_{r}$ created by a laser irradiance $I_{L}$ at the solid-vacuum interface is given by

$$
P_{r}=\left(\frac{I_{L}}{c}\right)(1+R)
$$

$R$ is the laser reflection from the micro-foil target and $c$ is the light velocity. For the following calculations, we take $R=1$ that might be achieved with very short laser pulses $\tau_{L}$ where $\tau_{L} \sim 10$ picoseconds and less. In this case, for a foil where the center of mass is moving in the $x$ direction, one gets from the Newton second law that the acceleration $a$ of the center of mass, in the $x$-direction, is given by

$$
a=\frac{2 I_{L}}{\rho c l_{x}} \equiv-g
$$

$\rho$ is the foil density and the $x, y, z$ dimensions of the foil are $l_{\mathrm{x}}$, $l_{\mathrm{y}}, l_{\mathrm{z}}$ accordingly. In this case, one can analyze the foil fluid motion in a gravitational field described by $g$. For a fluid motion described by its field velocities $v(x, y, z)$ without turbulence, $\nabla \times v=0$, one can define the velocity potential $\phi$ by the relation $v=\nabla \phi(x, y, z ; t)$. Neglecting the quadratic velocity term $\left(v^{2}\right)$ and assuming a non-compressible fluid one can write the Euler equation in a gravitational field in the following way

$$
\frac{\partial \phi}{\partial t}+\frac{P}{\rho}+g \mathrm{x}=0
$$

Using the relations $v_{x}=\partial x / \partial t=\partial \phi / \partial x, v_{y}=\partial \phi / \partial y, v_{z}=$ $\partial \phi / \partial z$, taking the local pressure $P$ as induced by the surface tension, $P=-\alpha\left(\partial^{2} x / \partial y^{2}+\partial^{2} x / \partial z^{2}\right)$, and taking the time derivative of Eq. (3) we get the following equation

$$
\rho g \frac{\partial \phi}{\partial x}+\rho \frac{\partial^{2} \phi}{\partial t^{2}}-\alpha \frac{\partial}{\partial x}\left(\frac{\partial^{2} \phi}{\partial y^{2}}+\frac{\partial^{2} \phi}{\partial z^{2}}\right)=0
$$

Defining the foil in the domain: $-l_{\underline{x}} \leq x \leq 0,0 \leq y \leq l_{y}, 0 \leq$ $z \leq l_{z}$, and assuming the boundary conditions

$$
\begin{aligned}
& \mathrm{v}_{x}\left(x=-l_{x}\right)=\frac{\partial \phi}{\partial x}\left(x=-l_{x}\right)=0 \\
& \mathrm{v}_{y}\left(y=0, l_{y}\right)=\frac{\partial \phi}{\partial y}(y=0)=\frac{\partial \phi}{\partial y}\left(y=l_{y}\right)=0, \\
& \mathrm{v}_{z}\left(z=0, l_{z}\right)=\frac{\partial \phi}{\partial z}(z=0)=\frac{\partial \phi}{\partial z}\left(z=l_{z}\right)=0
\end{aligned}
$$

we get the following solution of Eq. (4)

$$
\begin{aligned}
& \phi(x, y, z ; t)=e^{-i \omega t} A e^{-k l_{x}}\left[e^{k\left(x+l_{x}\right)}+e^{-k\left(x+l_{x}\right)}\right] f(y, z) \\
& \left.f(y, z)=\cos \left(\frac{n_{y} y}{l_{y}}\right) \cos \left(\frac{n_{z} z}{l_{z}}\right) ; \begin{array}{c}
n_{y} \\
n_{z}
\end{array}\right\}=0,1 \pi, 2 \pi, 3 \pi, \ldots
\end{aligned}
$$

Substituting this solution into Eq. (4) we get the following dispersion relation at the foil back surface $x=0$ (the laser irradiates the foil at the front surface, $x=-l_{x}$, while we are interested in the back surface instability about the surface $x=0)$.

$$
\omega^{2}=-k\left[a \cdot \operatorname{tgh}\left(k l_{x}\right)-\frac{\alpha}{\rho}\left(\frac{n_{y}^{2}}{l_{y}^{2}}+\frac{n_{z}^{2}}{l_{z}^{2}}\right)\right] .
$$

In Eq. (7), we have substituted the acceleration $a$ instead of the gravitational field $g$ ( $g=-a, a>0$ according to Eq. (2)).

Denoting by $\xi$ the local disturbance at the back surface of the foil we obtain from Eq. (6)

$$
\xi=\int\left(\frac{\partial \phi}{\partial x}\right)_{x=0} d t=\xi_{0} e^{-i \omega t}
$$

where $\xi_{0}$ is the initial disturbance at the $x=0$ surface and $\omega$ is given by Eq. (7). Neglecting the surface tension term (i.e., $\alpha=0$ ), $\omega$ is imaginary and one gets the RT instability by 
using Eq. (2)

$$
\begin{aligned}
& \frac{\xi}{\xi_{0}}=\exp \left\{\left[\mathrm{ka} \cdot \operatorname{tgh}\left(\mathrm{k} l_{\mathrm{x}}\right)\right]^{1 / 2} \mathrm{t}\right\} \equiv \exp \left(\frac{t}{\tau_{R T}}\right) \\
& \frac{1}{\tau_{R T}} \equiv\left[\left(\frac{2 \pi a}{L}\right) \operatorname{tgh}\left(\frac{2 \pi l}{L}\right)\right]^{1 / 2}=\left[\left(\frac{4 \pi I_{L}}{\rho c l L}\right) \operatorname{tgh}\left(\frac{2 \pi l}{L}\right)\right]^{1 / 2} .
\end{aligned}
$$

In the second term of Eq. (9), the most restrictive constrain on the instability is taken by choosing $k=2 \pi / L$, where $L$ is the target dimension orthogonal to the $x$ amplitude. For $\operatorname{tgh}\left(k l_{x}\right)=1$ one gets the standard RT expression (Eliezer, 2002; Chandrasekhar, 1961). However in our case $L \sim l_{y}=$ $l_{z} \gg l_{x} \equiv l$ implying $\operatorname{tgh}(2 \pi l / L) \sim 2 \pi l / L$ and the following time scale for the RT instability

$$
\tau_{R T}=\left(\frac{L}{2 \pi}\right) \sqrt{\frac{\rho c}{2 I_{L}}} \text { for } l \ll \mathrm{L}
$$

Denoting the critical value that the foil breaks by $\left(\xi / \xi_{0}\right)_{c r}$ at time $t_{c r}$, one gets the foil velocity $v_{f}$ before breakdown by RT instability

$$
\begin{aligned}
& \mathrm{v}_{f}=a t_{c r}=a \tau_{R T} \ln \left(\frac{\xi}{\xi_{0}}\right)_{c r} \Rightarrow \\
& \mathrm{v}_{f}=c\left\{\left(\frac{I_{L}}{\rho c^{3}}\right)\left(\frac{L}{\pi l}\right)\left[\operatorname{tgh}\left(\frac{2 \pi l}{L}\right)\right]^{-1}\right\}^{1 / 2} \ln \left(\frac{\xi}{\xi_{0}}\right)_{c r} .
\end{aligned}
$$

For $I_{L}=10^{18} \mathrm{~W} / \mathrm{cm}^{2}, L=10 \mu \mathrm{m}, l=1 \mu \mathrm{m}$, and $\rho=1 \mathrm{~g} / \mathrm{cm}^{3}$ we get using Eqs. (2) and (9) the acceleration $a=(2 / 3) \times$ $10^{19} \mathrm{~cm} / \mathrm{s}^{2}$ and the RT time scale $\tau_{\mathrm{RT}}=6.54 \mathrm{ps}$. This interesting result implies that a foil with very small initial disturbances can be accelerated until it breaks at $\ln \left(\xi / \xi_{0}\right)_{c r} \sim 3$ inferring $t_{c r} \sim 20$ ps before breaking down by RT instability. In this case, the foil velocity $v_{f}$ is about $v_{f} \sim 3 a \tau_{\mathrm{RT}}=1.34 \times$ $10^{8} \mathrm{~cm} / \mathrm{s}$.

The final velocity of the accelerated foil before breakdown by Rayleigh Taylor instability according to Eq. (11) is given in Figure 1, where we have chosen $\ln \left(\xi / \xi_{0}\right)_{c r}=3$ and the foil density $\rho=1 \mathrm{~g} / \mathrm{cm}^{3}$. The ratio of foil thickness $(l)$ to the transversal dimension $(L)$ are given for (1) $2 \pi l / L=1$, (2) $2 \pi l / L=0.1$. As one can see from these figures the laser can accelerate foils to velocities as high as $10^{9} \mathrm{~cm} / \mathrm{s}$ before breaking by RT instability.

\section{THE SURFACE TENSION AND RAYLEIGH-TAYLOR (RT) INSTABILITY}

In this section, we describe the double layer by a capacitor model as given in Figure 2. This capacitor model is controlled by laser irradiances $I_{L}$ where the ponderomotive force dominates the interaction. $E_{x}$ is the electric field inside the double layer, $\lambda_{\mathrm{DL}}$ is the distance between the positive and negative DL charges, $l$ is the foil thickness, and $\delta$ is the solid density skin depth of the foil. $n_{e}$ and $n_{i}$ are the
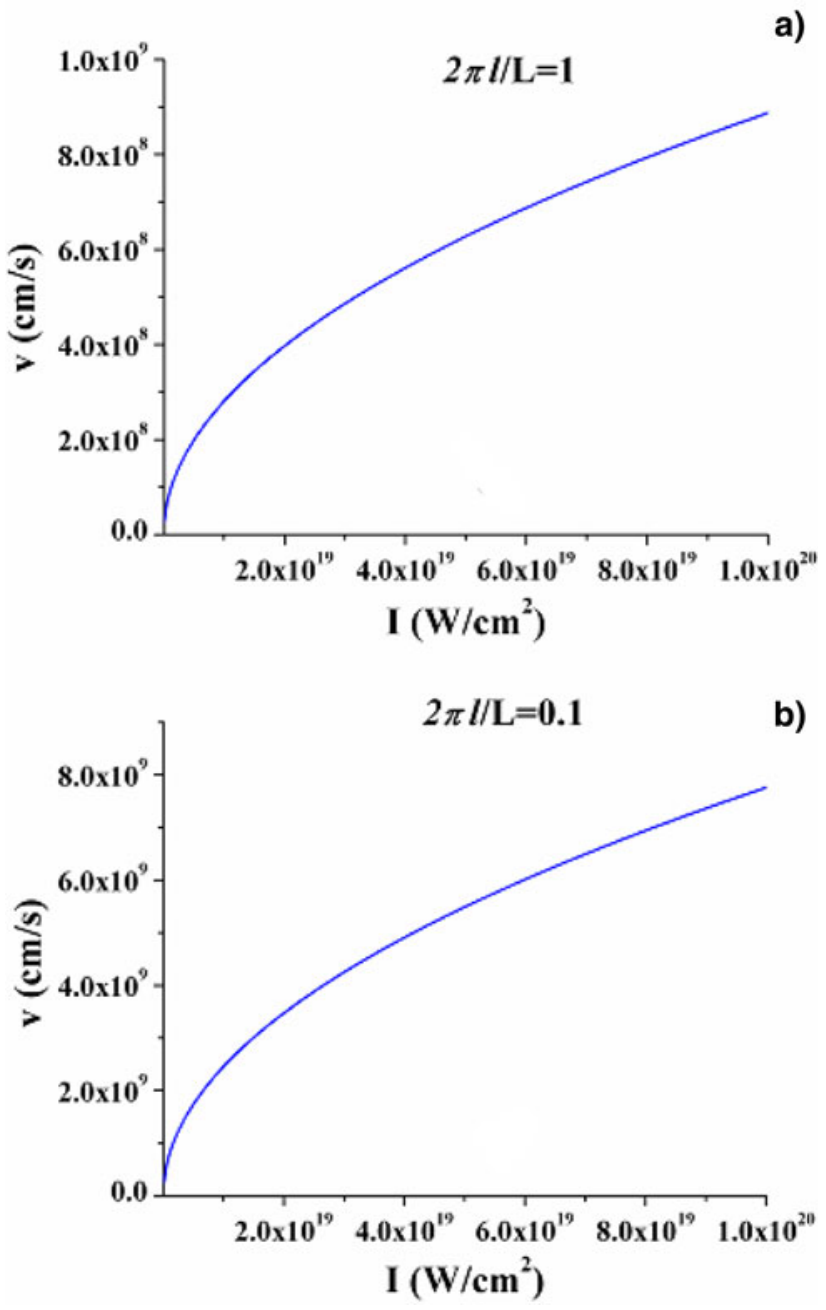

Fig. 1. (Color online) The final velocity of the accelerated foil before breakdown by Rayleigh Taylor instability according to Eq. (11). In this equation $\ln \left(\xi / \xi_{0}\right)_{c r}$ was chosen to be equal to 3 and the foil density $\rho=1 \mathrm{~g} / \mathrm{cm}^{3}$. The ratio of foil thickness $(l)$ to the transversal dimension $(L)$ are chosen for (a) $2 \pi l / L=1$, (b) $2 \pi l / L=0.1$.

electron and ion densities accordingly, $E_{x}$ is the electric field solution. The DL is geometrically followed by neutral plasma where the electric field decays within a skin depth $\delta$ given by $\delta=\mathrm{c} / \omega_{p e}$, where $\omega_{p e}=5.64 \times 10^{4} \sqrt{ } n_{e}=1.78 \times$ $10^{16} \mathrm{~s}^{-1}$, implying $\delta \sim 2 \times 10^{-6} \mathrm{~cm}$.

The acceleration of double layers by a high power laser to high velocity was suggested in the literature for a long time. The dispersion relation that takes into account also the double layer (DL) surface tension (Eliezer \& Hora, 1989; Hora et al., 1989) is given in Eq. (7). The double layer motion is RT stable if $\omega^{2}>0$ and this can be achieved if the following inequality is satisfied:

$$
a \geq \rho a L^{2} \cdot \operatorname{tgh}\left(\frac{2 \pi l}{L}\right)
$$

In Eq. (12), we have taken the most unstable modes as defined in Eq. (7) by $\left(n_{y}=2 \pi, n_{z}=0\right)$ or $\left(n_{y}=0, n_{z}=2 \pi\right)$. 


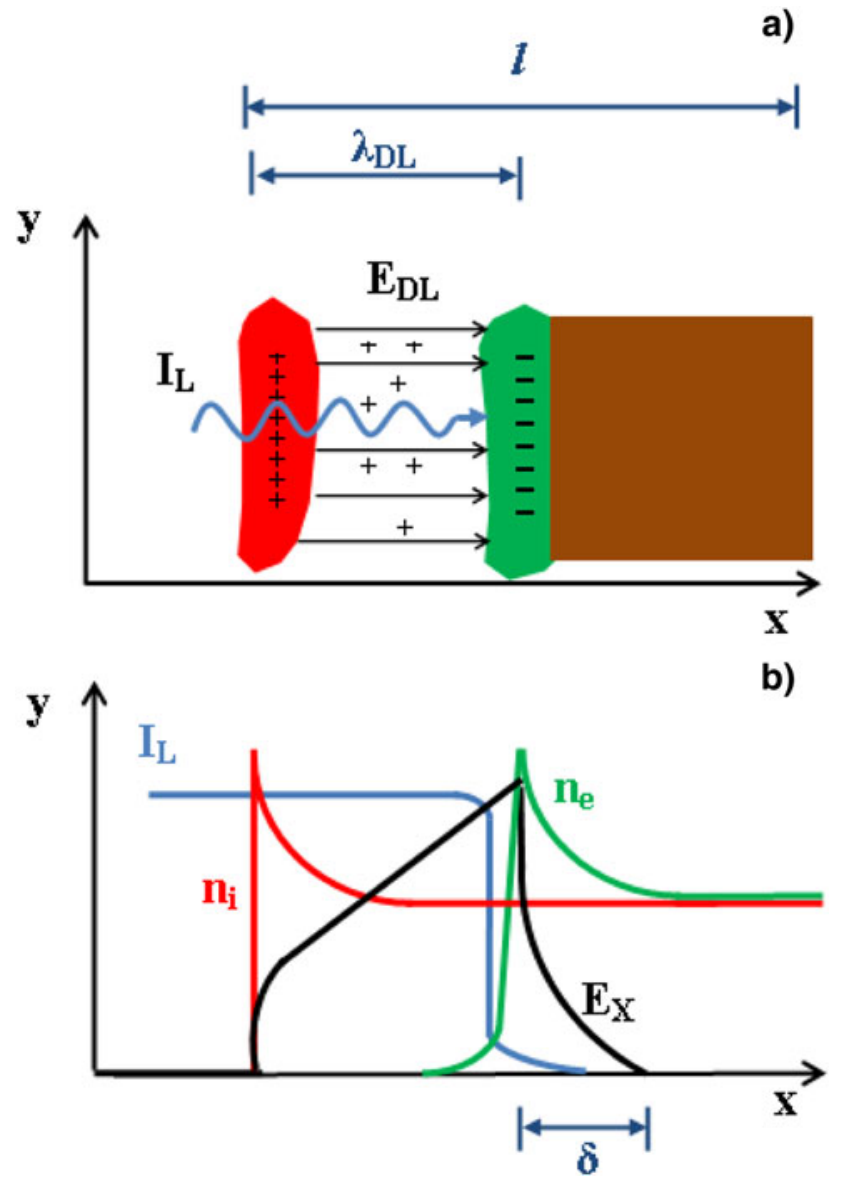

Fig. 2. (Color online) (a) The capacitor model for laser irradiances $I_{L}$ where the ponderomotive force dominates the interaction. $E_{x}$ is the electric field inside the double layer, $\lambda_{\mathrm{DL}}$ is the distance between the positive and negative DL charges, $l$ is the foil thickness and $\delta$ is the solid density skin depth of the foil. (b) A schematic figure that our capacitor model is based. $n_{e}$ and $n_{i}$ are the electron and ion densities accordingly, $E_{x}$ is the electric field solution. The DL is geometrically followed by neutral plasma where the electric field decays within a skin depth.

The DL surface tension within the context of a capacitor model (see Fig. 2) is given by

$$
\alpha=\left(\frac{E_{D L}^{2}}{8 \pi}\right) \lambda_{D L} \approx \frac{I_{L} \lambda_{D L}}{c \varepsilon_{r}^{2}}
$$

$E_{\mathrm{DL}}$ is the electric field in the DL region; $\lambda_{\mathrm{DL}}$ is the distance between the positive and negative DL charges, usually (Eliezer \& Hora, 1989) of the order of the Debye length $\lambda_{\mathrm{D}} \approx$ $750\left(T_{\mathrm{eV}} / n_{e}\right)^{1 / 2}$ where $T_{\mathrm{eV}}$ and $n_{e}$ are the electron temperature in $\mathrm{eV}$ units and the electron density accordingly. $\varepsilon_{r} \approx(1-$ $\left.\omega_{p}^{2} / \omega_{L}^{2}\right)=\left(1-n_{e} / n_{c}\right)$ is the dielectric constant in the DL domain. Since the electrons are pushed out of the DL by the ponderomotive force then one can assume that the electron density inside the DL is smaller than the critical density $n_{c}$, described schematically in Figure 2 , implying $\varepsilon_{r} \approx 1$ as a good approximation. Substituting the acceleration from
Eq. (2) into Eq. (13) we get the DL stability for

$$
\lambda_{D L} \geq 2\left(\frac{L^{2}}{l}\right) \operatorname{tgh}\left(\frac{2 \pi l}{L}\right) .
$$

For $l / L \ll 1$ we can approximate the DL stability by the simple relation

$$
\lambda_{D L} \geq 4 \pi L
$$

As a typical example we can take $\lambda_{\mathrm{DL}}$ of the order of the Debye length $\lambda_{\mathrm{D}}$ and for $T=100 \mathrm{eV}, n_{e}=10^{22} \mathrm{~cm}^{-3}$ we have $\lambda_{\mathrm{DL}} \sim 7.5 \times 10^{-8} \mathrm{~cm} \sim 10 \mathrm{~L}$, a transversal dimension of a nano-foil or rather a nanotube! Therefore it looks that for micro-foils the DL surface tension does not contribute to hydrodynamic stability unless the electron density is few orders of magnitude smaller than $10^{22} \mathrm{~cm}^{-3}$ and the temperature significantly higher than $100 \mathrm{eV}$.

\section{RELATIVISTIC RAYLEIGH-TAYLOR (RT) INSTABILITY}

With the development (Piazza et al., 2012) of laser irradiances larger than $10^{21} \mathrm{~W} / \mathrm{cm}^{2}$ it became possible to accelerate a micro-foil to relativistic velocities due to the laser pressure $P=2 I_{F} / c$, where $I_{F}$ is the laser irradiance in the accelerated foil rest frame and $c$ is the speed of light and it is assumed that the reflection in the foil rest frame is one. The laser irradiance $I_{L}$ and angular frequency $\omega_{L}$ in the laboratory are related to their appropriate values in the foil rest frame (denoted by subscript $F$ ) by Doppler equation, based on appropriate Lorenz transformation of the electro-magnetic fields and the definition of $I$ in term of these fields,

$$
\begin{aligned}
& I_{L}=I_{F}\left(\frac{\omega_{L}}{\omega_{F}}\right)^{2}=I_{F}\left(\frac{1+\beta_{f}}{1-\beta_{f}}\right) \\
& P=\frac{2 I_{L}}{c}\left(\frac{1-\beta_{f}}{1+\beta_{f}}\right)
\end{aligned}
$$

where $c \beta_{f}$ is the foil velocity in the laboratory frame of reference.

The relativistic Newton law of motion

$$
\frac{d}{d t}\left[\left(\rho_{0} l c\right) \frac{\beta_{f}}{\sqrt{1-\beta_{f}^{2}}}\right]=\frac{2 I_{L}}{c}\left(\frac{1-\beta_{f}}{1+\beta_{f}}\right),
$$

has been solved (Eliezer et al., 2013)

$$
\int_{0}^{\beta_{f}} \frac{d x}{(1-x)^{5 / 2}(1+x)^{1 / 2}}=\frac{\left(2-\beta_{f}\right) \sqrt{1-\beta_{f}^{2}}}{3\left(1-\beta_{f}\right)^{2}}-\frac{2}{3}=\frac{2 I t}{\rho_{0} c^{2} l} \equiv \frac{t}{\tau},
$$


for a pulsed laser with constant intensity in order to get micro-foil velocity $\beta_{f}$ as function of the laser pulse duration $t_{L}$ in units of $\tau=\rho_{0} c^{2} l /\left(2 I_{L}\right)$, where $\rho_{0}$ is the micro-foil initial density and $l$ is the foil thickness. Figure 3 describes the micro-foil velocity as a function of the laser pulse duration in units of $\tau$. For example, if $\rho_{0}=1 \mathrm{~g} / \mathrm{cm}^{3}, l=0.1 \mu \mathrm{m}$, foil cross-section of $10 \mu \mathrm{m}^{2}$, then $\beta_{f}=0.5$ for a laser with $I_{L} t_{L}=4.5 \times 10^{8} \mathrm{~J} / \mathrm{cm}^{2}$ and energy $45 \mathrm{~J}$. Furthermore, for an irradiance of $10^{22} \mathrm{~W} / \mathrm{cm}^{2}$ one requires a laser with pulse duration of $45 \mathrm{fs}$.

A crucial question in accelerating a foil to relativistic velocity is its hydrodynamic stability. In particular, the relativistic Rayleigh-Taylor instability was calculated (Pegoraro \& Bulanov, 2007) as described in Eq. (19).

$$
\begin{aligned}
& \xi_{N R}=\frac{\Delta x}{x_{0}}=\exp \left(\frac{t}{\tau_{N R}}\right) \\
& \xi_{R}=\frac{\Delta x}{x_{0}}=\exp \left[\left(\frac{t}{\tau_{R}}\right)^{1 / 3}\right] \\
& \frac{\tau_{R}}{\tau_{N R}}=\left(\frac{1}{3 \pi}\right)\left(\frac{L}{l_{0}}\right)\left(\frac{I_{L}}{\rho_{0} c^{3}}\right)
\end{aligned}
$$

$\xi_{\mathrm{NR}}$ and $\xi_{\mathrm{R}}$ are accordingly the non-relativistic and relativistic development of the instability for an initial disturbance $x_{0}$ and $L$ is the target dimension orthogonal to the $x$ amplitude. The non-relativistic time scale $\tau_{N R}$ we calculate from the second of Eq. (9): $\tau_{N R}=\tau_{\mathrm{RT}}$. We consider the following example: $L=10 \mu \mathrm{m}, x_{0}=10 \mathrm{~nm}, \rho_{0}=1 \mathrm{~g} / \mathrm{cm}^{3}, l=0.1 \mu \mathrm{m}$, $I_{L}=10^{24} \mathrm{~W} / \mathrm{cm}^{2}$. Assuming that the foil breaks for $\xi=10$ (i.e., $\Delta x \sim l)$ then the foil breaks at $14.2 \mathrm{fs}\left(\tau_{N R}=6.2 \mathrm{fs}\right)$ for the non-relativistic case while in the relativistic regime the foil is stable during $90.5 \mathrm{fs}\left(\tau_{R}=39.3 \mathrm{fs}\right)$. This behavior

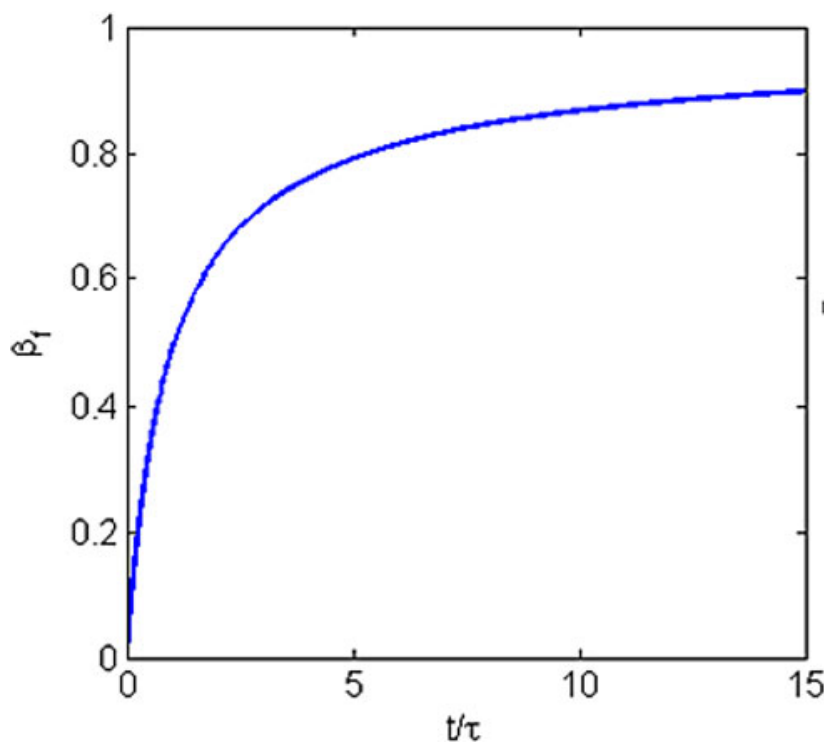

Fig. 3. (Color online) Micro-foil velocity $\beta_{f} c$ as a function of the laser pulse duration in units of $\tau=\rho_{0} c^{2} l /\left(2 I_{L}\right)$, where $\rho_{0}$ is the micro-foil initial velocity upon impact and $l$ is the foil thickness. is understood from the different time dependence of the RT instability in relativistic and non-relativistic cases. Taking into account that during few femtoseconds one can accelerate a micro-foil to relativistic velocities one can achieve stable relativistic acceleration for laser irradiances of the order of $10^{23} \mathrm{~W} / \mathrm{cm}^{2}$.

\section{DISCUSSION}

In this paper, we analyze the acceleration of a micro-foil by the laser radiation pressure impact. We look for acceleration without laser-plasma ablation. The laser irradiances of interest are in the domain $10^{17} \mathrm{~W} / \mathrm{cm}^{2}<I_{L}<10^{21} \mathrm{~W} / \mathrm{cm}^{2}$ for non-relativistic acceleration and $I_{L} \geq 10^{21} \mathrm{~W} / \mathrm{cm}^{2}$ for the relativistic regime. Due to the nature of the ponderomotive force, in both the relativistic and non-relativistic cases, the structure of the accelerated target contains a double layer as shown in Figure 2. Furthermore, for ultra-high irradiance lasers the RT instability does not apparently disturbs the acceleration as has been derived theoretically in particle in cell simulations (Esirkepov et al., 2004) of a foil and two fluid hydrodynamic simulations of plasma blocks (Lalousis et al., 2013; 2012; Hora et al., 2014).

The main result of this research is that the laser radiation pressure can accelerate a micro-foil to very high velocities without breaking it by the RT instabilities. For the nonrelativistic domain the laser time duration is of the order of $\tau_{L} \sim 10$ picoseconds while in the relativistic case the laser pulse is $\tau_{L} \sim 10$ femtoseconds.

The surface tension is also analyzed using a capacitor model in the context of hydrodynamic stability. It is shown that for solid state density the surface tension cannot stabilize a micro-foil but it might cause stabilization for nano-foils not discussed in this paper (Eliezer et al., 2004; 2005). However, for under dense DL plasmas, the surface tension can be an important stabilizing mechanism.

The non-relativistic velocities that can be achieved are given explicitly in Eq. (11) and described in Figures 1. As one can see from these figures the laser can accelerate foils to velocities as high as $10^{9} \mathrm{~cm} / \mathrm{s}$ before breaking by RT instability. The relativistic velocities are specified implicitly in Eq. (18) as designated in Figure 3. Therefore, for the state of the art high irradiance lasers the RT instability can be avoided during the acceleration time and relativistic velocities can be achieved.

Finally, we would like to suggest that the consequences of this paper may be important in discussing the possibility of fast ignition (Basov et al., 1992; Tabak et al., 1994) nuclear fusion triggered by laser or by foil impact induced shockwaves (Betti et al., 2007; Eliezer \& Martinez Val, 2011; Murakami et al., 2006; Eliezer et al., 2007). The "fast ignition" idea is to ignite the fuel (e.g., deuterium- tritium, proton-boron 11 , etc.) with less energy and in order to achieve this goal one has to separate the drivers that compress and ignite the target. First the fuel is compressed then a second driver ignites a small part of the fuel while the created 
alpha particles heat the rest of the target. The fast ignition problem is that the laser pulse does not penetrate directly into the compressed target; therefore many schemes have been suggested. In this context the comeback of shock waves in inertial fusion energy was published and the ignition by impact was suggested (Eliezer, 2012; Murakami et al., 2006). The RT instability plays a crucial role in achieving ignition (Mima et al., 2009) and therefore the analysis of this paper might be important.

\section{REFERENCES}

Azechi, H., Sakaiya, T., Watari, T., Karasik, M., Saito, H., Ohtani, K., Takeda, K., Hosoda, H., Shiraga, H., Nakai, M., Shigemori, K., Fujioka, S., Murakami, M., Nagatomo, H., Johzaki, T., Gardner, J., Colombant, D.G., Bates, J.W., Velikovich, A.L., Aglitskiy, Y., Weaver, J., Obenschain, S., Eliezer, S., Kodama, R., Norimatsu, T., FujtTa, H., Mima, K. \& Kan, H., (2009). Experimental evidence of impact ignition: 100-fold increase of neutron yield by impactor collision. Phys. Rev. Lett. 102, 235002.

Basov, N.G., Guskov, S.Y. \& Feoktistov, L.P. (1992). Thermonuclear gain of ICF targets with direct heating of the ignitor. J. Soviet Laser Res. 13, 396.

Betti, R., Zhou, C.D., Anderson, K.S., Perkins, L.J., Theobald, W. \& Sokolov, A. A. (2007). Shock ignition of thermonuclear fuel with high areal density. Phys. Rev. Lett. 98, 155001.

Cauble, R., Phillion, D.W., Hoover, T.J., Holmes, N.C., Kilkenny, J.D. \& LEE, R.W. (1993). Demonstration of 0.75 Gbar planar shocks in x-ray driven colliding foils. Phys. Rev. Lett. 70, 2102.

Chandrasekhar, S. (1961) Hydrodynamic and Hydromagnetic Stability. Oxford: Oxford University Press.

Clark, P.J., Eliezer, S., Farley, F.J.M., et al. (1985). Laser focus accelerator by relativistic self-focusing and high electric fields in double layers of nonlinear force produced cavitons. AIP Conf. Proc. 130, 380.

Eliezer, S. \& HorA, H. (1989). Double layers in laser produced plasmas. Phys. Repts 172, 339.

Eliezer, S. (2002). The Interaction of High Power Lasers with Plasmas. Boca Raton: CRC press.

Eliezer, S., Eliaz, N., Grossman, E. et al. (2004). Synthesis of nanoparticles with femtosecond laser pulses. Phys. Rev. B 69, 144119.

Eliezer, S., Eliaz, N., Grossman, E., Fisher, D., Gouzman, I., Henis, Z., Pecker, S., Horovitz, Y., Fraenkel, M., Maman, S. \& Lereah, Y. (2005). Nanoparticles and nanotubes induced by femtosecond lasers. Laser Part. Beams 23, 15.
Eliezer, S., Murakami, M. \& Martinez Val, J.M. (2007). Equation of state and optimum compression in inertial fusion energy. Laser Part. Beams 25, 1.

Eliezer, S. \& Martinez VAL, J.M. (2011). The comeback of shock waves in inertial fusion energy. Laser Part. Beams 29, 175.

ELIEZER, S. (2012). Relativistic acceleration of micro-foils with prospects for fast ignition. Laser Part. Beams 30, 225.

Eliezer, S., Martinez Val, J.M. \& Pinhasi, S.V. (2013). Relativistic shock waves in the laboratory. Laser Part. Beams 31, 113.

Esirkepov, T., Borghesi, M., Bulanov, S.V., Mourou, G. \& Tajima, T. (2004). Highly efficient relativistic ion generation in the laser-piston regime. Phys. Rev. Lett. 92, 175003.

Hora, H., Lalousis, P. \& Eliezer, S. (1984). Analysis of the inverted double layers produced by nonlinear forces in laser produced plasmas. Phys. Rev Lett. 53, 1650.

Hora, H., Min, G., Eliezer, S., Lalousis, P., Pease, R.S. \& SzichMAN, H. (1989). On the surface tension of plasmas. IEEE Trans. Plasma Sci. 17, 283.

Hora, H., Lalousis, P.\& Moustaizis, S. (2014). Picosecond laser pulses for ultrahigh plasma acceleration to ignite fusion flames in solid density fuels. IFSA 2013 Proceedings, Nara, Japan.

LALuOSIS, P., FoldEs, I.B. \& HorA, H. (2012). Ultrahigh acceleration of plasma by picosecond terawatt laser pulses for fast ignition of fusion. Laser Part. Beams 30, 233.

Lalousis, P., Hora, H., Eliezer, S., Martinez Val, J.M., MoustaIzIS, S., Miley, G. H. \& Mourou, G. (2013). Shock Mechanisms by ultrahigh laser accelerated plasma blocks in solid density targets for fusion. Phys. Lett. A 377, 885.

Mima, K., Murakami, M., Nakai, S. \& Eliezer, S. (2009). Applications of Laser-Plasma Interactions. Boca Raton: CRC Press.

Murakami, M., Nagatomo, H., Azechi, H., Ogando, F. \& Eliezer, S. (2006). Innovative ignition scheme for ICF impact fast ignition. Nucl. Fusion 46, 99.

Pegoraro, F. \& Bulanov, S.V. (2007). Photon bubbles and ion acceleration in a plasma dominated by the radiation pressure of an electromagnetic pulse. Phys. Rev. Lett. 99, 065002.

Piazza, A.D., Muller, C., Hatsagortsyan, K. Z. \& Keitel, C. H. (2012). Extremely high-intensity laser interactions with fundamental quantum systems. Rev. Mod. Phys. 84, 1177.

Rayleigh, J.W.S. Lord. (1876). On waves. Phil. Mag. 1, 257-279.

TabaK, M., Hammer, J., Glinsky, M.E., Kruer, W.L., Wilks, S.C., Woodworth, J., Campbell, E., Perry, M.D. \& Mason, R.J. (1994). Ignition and high gain with ultra-powerful lasers. Phys. Plasmas 1, 1626.

TAYLOR, G. (1950). The instability of liquid surfaces when accelerated in a direction perpendicular to their planes. Proc. Roy. Soc. London A 201, 192. 